\title{
Redeposition of amorphous hydrogenated carbon films during thermal decomposition
}

\author{
E. Salançon*, T. Dürbeck, T. Schwarz-Selinger, F. Genoese, Wolfgang Jacob ${ }^{\dagger}$ \\ Max-Planck-Institut für Plasmaphysik, EURATOM Association, Boltzmannstr. 2, 85748 Garching, Germany
}

\begin{abstract}
hermally induced decomposition of hard and soft amorphous hydrocarbon films was investigated by thermal effusion spectroscopy. Released species were detected by a sensitive quadrupole mass spectrometer using two different experimental setups for thermal effusion. Species released in a molecular beam setup were detected in direct line of sight to the sample surface, while species released in a remote UHV oven had no direct line of sight to the mass spectrometer. Soft, hydrogen-rich carbon films exhibit a desorption maximum at $\mathrm{T} \approx 740 \mathrm{~K}$ while hard films with a low hydrogen content have their maximum at $\mathrm{T} \approx 870 \mathrm{~K}$. Additionally, the spectrum of released species differs dramatically between hard and soft films. We found a significant redeposition of species released from soft films. From the redeposited fraction of material we estimated an average redeposition probability of about $50 \%$ for species released from soft films.
\end{abstract}

PACS numbers: 52.40.Hf, 68.60.Dv, 81.70.Pg, 28.52.Fa

Published in J. Nucl. Mater.: Received 02 August 2007

Revised version received 12 December 2007

Accepted for publication 27 January 2008

doi:10.1016/j.jnucmat.2008.02.070

J. Nucl. Mater. 376 (2008) 160-168

\section{INTRODUCTION}

Plasma-facing components in thermonuclear fusion devices have been mainly made of carbonaceous materials in the last years. In the present design for ITER it is also foreseen to build parts of the divertor-the strike zone-from CFC (carbon fiber composites) material $[1,2]$. These surfaces are exposed to a substantial incoming flux of ions and neutrals from the main plasma which leads to erosion of the divertor tiles emitting carbon and hydrocarbon compounds into the boundary plasma. Most of these species released in the divertor will redeposit in relative close proximity to their place of origin and this balance between erosion and deposition is crucial for the performance of a divertor. However, the small fraction of carbon and hydrocarbon species that is not redeposited in the divertor may escape from the divertor and also from the boundary plasma and cause deposition of hydrocarbon layers (often called co-deposited or redeposited layers) on surfaces not in direct contact with plasma.

A number of investigations conducted in fusion plasma devices as well as in laboratory experiments have been devoted to study the deposition of such redeposited layers [3-11]. Redeposited layers growing in remote areas without direct plasma contact are soft hydrocarbon films with high hydrogen content and it seems that hydrocarbon species with a relatively high sticking coefficient contribute dominantly to deposition [12]. A major concern for future fusion devices such as ITER is the large amount of hydrogen isotopes trapped in these redeposited films because then this trapped hydrogen will partly be tritium $[1,11]$. Techniques to remove hydrogen isotopes

* Present address: Physique des Interactions Ioniques et Moléculaires, CNRS and Université de Provence (UMR 6633), Campus Universitaire de St Jérôme service 241, 13397 Marseille cedex 20, France

${ }^{\dagger}$ E-mail: from fusion devices were recently reviewed by Counsell et al. [11]. Among others, heating of redeposited films in vacuum either with a rapidly scanning laser beam $[13,14]$ or with a flash lamp $[15,16]$ was proposed as a possible method to thermally desorb tritium. One problem of this method is that little is known about the product spectrum of the released hydrocarbon species. E.g., it is a priori not clear whether all of the released products are stable, non-sticking species which can be transported to the pumps and such finally be removed from the vacuum vessel. This was already briefly discussed by Gibson et al. [15]. Species with a high sticking coefficient may be volatilized at one place, but may redeposit somewhere else on the wall before reaching the pump so that they are not removed from the vacuum vessel.

In this article we present thermal effusion spectra of two different types of amorphous hydrocarbon films deposited in low-temperature laboratory plasmas. These films are a model system for redeposited carbon layers in fusion devices. We determine the desorption temperatures, the spectrum of released species and the amount of redeposited material for both types of films.

\section{EXPERIMENTAL}

\section{A. Thermal effusion measurements}

Thermal effusion spectra are measured in the experimental device TESS (Thermal Effusion Spectroscopy Setup) specifically designed to allow sensitive thermal effusion studies of thin films and surface layers. A schematic drawing of the experimental setup is shown in Fig. 1. TESS is an ultrahigh vacuum (UHV) experiment equipped with a cryopump to provide high pumping speed and a sensitive quadrupole mass spectrometer (QMS).

Additionally, the main chamber is equipped with a liquid nitrogen trap located below the sample holder (see Fig. 1) to 

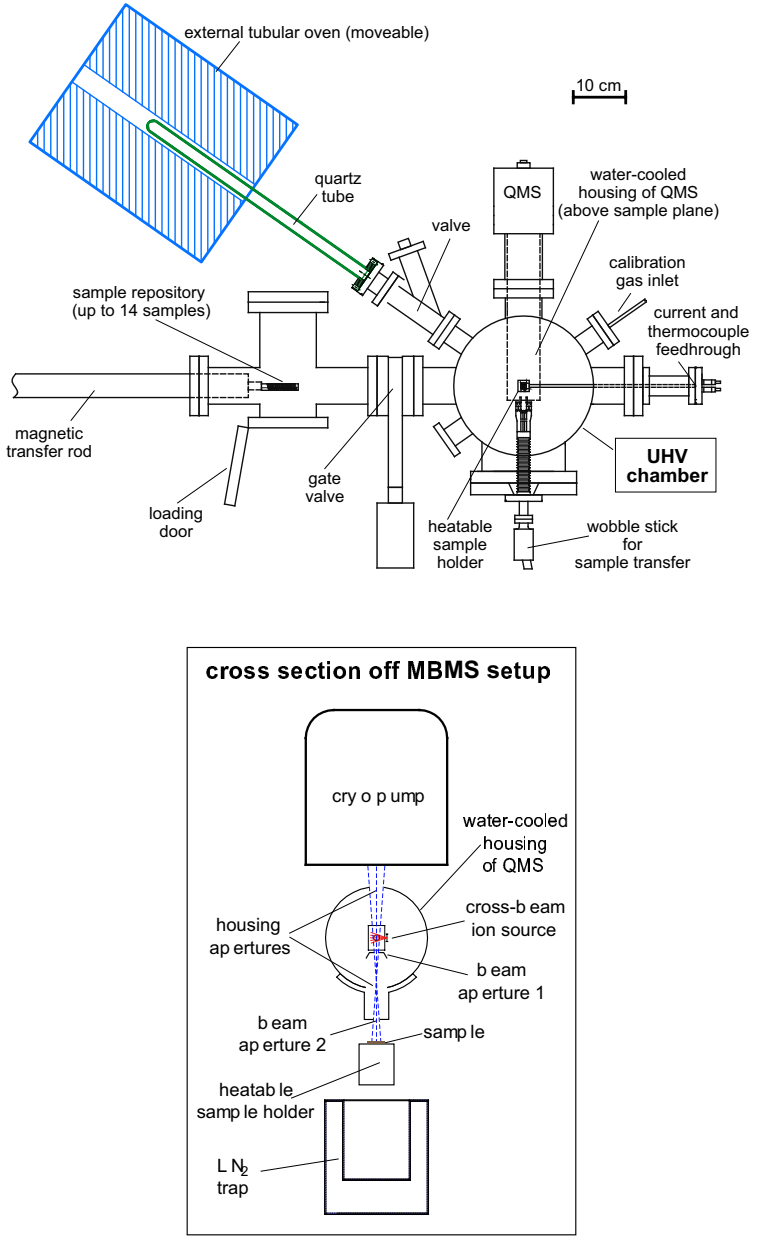

FIG. 1: Sketch of the experimental setup for thermal effusion measurements (TESS). The figure on the right-hand side shows a cross section of the molecular-beam mass spectrometry (MBMS) setup in the UHV chamber.

reduce the pressure of recycling species during thermal effusion experiments. A cold cathode gauge was used during thermal effusion runs to record the total pressure in the system. We checked that the operation of the cold cathode gauge produces no additional species in the mass spectra. The base pressure of TESS is in the upper $10^{-9} \mathrm{~Pa}$ range.

The Quadrupole Mass Spectrometer is a Pfeiffer/Inficon DMM 422 equipped with a cross-beam ion source. The quadrupole device is contained in a water-cooled housing to minimize the influence of heating onto the QMS system during effusion runs. The whole setup is designed such that species desorbing from the sample reach the ionizer of the mass spectrometer in direct line of sight. With other words, desorbing species are detected in a molecular beam geometry (MBMS = molecular beam mass spectrometry) which allows to measure reactive and non-reactive species with comparable sensitivity. The QMS housing is not differentially pumped so that in addition to the species in the molecular beam species recycling at the chamber walls can also reach the ionizer and thus contribute to the signal.

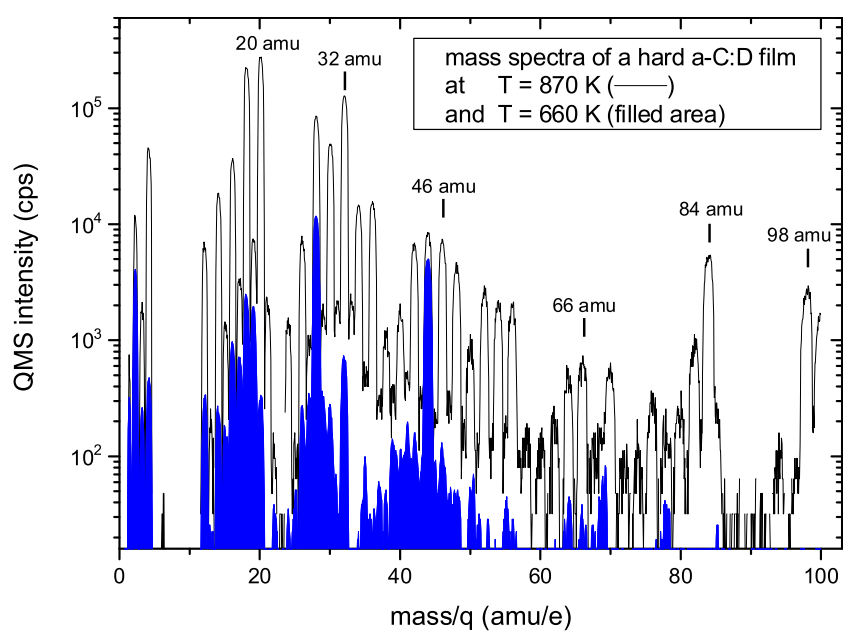

FIG. 2: Mass spectra in the range from 1 to $100 \mathrm{amu} / \mathrm{e}$ measured during a $\mathrm{TE}$ run. The spectrum at $\mathrm{T}=660 \mathrm{~K}$ is comparable to the residual background. The spectrum at $\mathrm{T}=870 \mathrm{~K}$ corresponds to the maximum of the effusion flux. The temperature rise during one mass scan is less than $20 \mathrm{~K}$.

Thermal effusion spectra were measured in two complimentary modes. To obtain an overview over the diversity of released species we recorded quasi continuous mass spectra (16 steps per amu) in the range from 1 to $100 \mathrm{amu}$ while the temperature of the sample was ramped. An example of such data is shown in Fig. 2. It shows the measured QMS intensity in a mass range from 1 to 100 amu at two selected temperatures during a thermal effusion run (TE run) of a hard a-C:D film. The spectrum measured at $\mathrm{T}=660 \mathrm{~K}$ is slightly higher but still comparable with a background spectrum prior to starting the temperature ramp. At this temperature, thermal effusion has not yet started. The spectrum measured at $\mathrm{T}=870 \mathrm{~K}$ corresponds to the maximum of the effusion flux. We find a strong increase of the QMS signal for almost all mass channels. Prominent peaks are 20,32, 44, 84, and $98 \mathrm{amu}$. The interpretation of these data is discussed further below. This mode yields the complete information about released species results, however, in a low time respectively temperature resolution. For the spectra shown in Fig. 2 the temperature rise during one complete mass spectrum, i.e., the temperature resolution, is about $20 \mathrm{~K}$. Alternatively, up to 64 mass channels were measured as a function of time while the sample temperature was ramped up. After the experiment the measured temperatures were calibrated and converted to the true sample temperature as described further below. This allowed us to convert the time axis into a temperature axis. This procedure yields the thermal effusion spectra as a function of temperature shown in this article. The temperature resolution in this mode is of the order of 2 to $3 \mathrm{~K}$ (depending on number of mass channels measured and experimental settings), that means it is about an order of magnitude better than in the other mode.

Samples are introduced into the main chamber via a load lock. In the load lock we have a sample magazine which can store up to 14 samples, so that, on the one hand, we are able 
to store samples in vacuum and, on the other hand, we do not have to open the load lock for each sample transfer. This assures that the base vacuum in the main chamber after sample transfer is not seriously impaired.

Samples are placed on a molybdenum sample holder in the main chamber with a wobble stick. Prior to a thermal effusion experiment the sample holder is cleaned by heating it up to $1300 \mathrm{~K}$ for one hour to minimize background contributions from the heater and the surrounding surfaces. The sample holder is heated from the backside by radiation from a resistively heated tantalum filament. It is shielded by a stack of heat shields to reduce heating of the surrounding surfaces. The temperature of the molybdenum block is measured by a Chromel/Alumel ( $\mathrm{Ni} / \mathrm{NiCr})$ thermocouple. It allows generating a linear temperature ramp controlled by a PID controller (Eurotherm 902P). The temperature ramp used in the experiments presented here is $15 \mathrm{~K} / \mathrm{min}$. Since the samples are put onto the heater plate without additional clamping, the thermal contact between sample and heater plate is low. Specific measures which have been taken to determine the true surface temperature of the samples are described further below.

In addition to this MBMS setup, TESS comprises a remote UHV oven. Released species are detected with the very same QMS as in the MBMS setup which allows a quantitative comparison of the measured mass spectra. The oven consists of a long quartz glass tube inserted into an external tubular oven. The heated volume of the oven is $4 \mathrm{~cm}$ in diameter and $40 \mathrm{~cm}$ in length. The external oven is mounted on a rail system and can be moved over the entire length of the quartz tube. It can also be removed completely from the quartz tube. The length of this quartz glass tube is $45 \mathrm{~cm}$ and its inner diameter is $2.0 \mathrm{~cm}$ (outer diam. $=2.54 \mathrm{~cm}$ ). The glass tube is connected to the main chamber via a gate valve. Sample loading is done by removing the tube. Several samples can be stored in the glass tube. After mounting the glass tube back to the vacuum system, it is pumped via a second gate valve through the load lock to a sufficiently low pressure (better than $10^{-4} \mathrm{~Pa}$ ). Only for measurement of effusion spectra the gate valve to the main chamber is opened. The background pressure in the main chamber during a measurement with the oven setup is in general $2 \times 10^{-8} \mathrm{~Pa}$. Samples are stored at the end of the glass tube which is connected to the main chamber. They can be manipulated in vacuum with a piece of nickel inside the tube which in turn is manipulated by a magnet outside the tube. For an effusion measurement the desired sample is moved to a position about $10 \mathrm{~mm}$ from the closed end of the tube and the external oven is centered around this location. The distance between the measurement position and the storage position is about $40 \mathrm{~cm}$. This means that the distance of the samples in the storage position from the end of the hot zone is about $15-20 \mathrm{~cm}$. Due to the low heat conductivity of quartz, the wall temperature of the quartz tube falls to room temperature within less than $1 \mathrm{~cm}$ outside the oven region, so that the samples in storage position always stay at room temperature. Prior to an experiment in the oven, the measurement region is heated to $1300 \mathrm{~K}$ for one hour to thoroughly clean the glass tube and to reduce background contributions from the walls of the glass tube during the measurement. The sample is trans- ferred from the storage position to the measurement position after the tube has cooled to about room temperature and then the measurement is immediately started.

Species released in this quartz glass oven reach the ionizer of the QMS only after many collisions with the walls of the quartz tube and the main vacuum chamber, so that reactive species which are lost in wall collisions, cannot reach the ionizer. With this setup only stable, non-reactive species can be detected. From a comparison of experiments performed in the MBMS and quartz glass oven setups information on the fraction of released reactive ('sticking') species can be extracted.

\section{B. Temperature calibration}

\section{Oven setup}

The oven temperature is controlled by an external temperature controller (Eurotherm 902P). It can provide heating rates of up to $30 \mathrm{~K} / \mathrm{min}$, but in the experiments presented here we used a temperature ramp of $15 \mathrm{~K} / \mathrm{min}$.

The temperature profile around the sample position for the usual experimental configuration was measured by fixing a thermocouple to a test sample which was placed at the sample position inside the quartz glass tube. For a set temperature of $1275 \mathrm{~K}$, the resulting true sample temperature is $1211 \mathrm{~K}$. The difference between the set temperature and the true sample temperature depends on the applied heating rate. We carefully measured calibration curves for the sample temperature as a function of the oven temperature and checked the reproducibility of this procedure regularly. The reproducibility is excellent and the uncertainty of the temperature determination in this setup is estimated to $\pm 5 \mathrm{~K}$. A big advantage of the quartz oven setup is that the samples are embedded in a homogeneous radiation field which can be very well controlled. This guarantees identical heating rates and temperatures in different experimental runs using the same experimental settings.

\section{MBMS setup}

The precise determination of the sample surface temperature in the MBMS setup is challenging because, in contrast to the quartz glass oven, the sample is not in a homogeneous radiation field. What is controlled during the experiment is the temperature of the molybdenum substrate holder. At low temperature, the heat transfer is dominantly due to thermal conduction. But the thermal contact between holder and sample is not well defined so that the temperature of the sample is in principal not well known. At high temperature, heat transfer is dominated by radiation and the thermal contact to the heater surface is less important. We followed two complementary strategies to determine the real sample temperatures.

First, we used a pyrometer specifically designed for the use with silicon (Impac IS $10 \mathrm{Si}$ ) to measure the sample temperature. This device uses a small-band infrared filter with a measurement range of about $0.95 \pm 0.03 \mu \mathrm{m}$. In this wavelength 
range the emissivity of silicon shows no pronounced temperature dependence and remains relatively constant at 0.67 . The operating range of this pyrometer is 650 to $1600 \mathrm{~K}$. The measurement area as defined by the beam optics is about 3-4 $\mathrm{mm}$ in diameter. The pyrometer is directed from above onto the sample surface under an angle of about $70^{\circ}$.

The samples we are interested in are in general coated with a thin hydrocarbon film. Properties of this surface layer change as a function of annealing temperature. Consequently, the emissivity of this surface layer also changes as a function of temperature, so that measured temperatures are rather uncertain. We made a number of test experiments using coated and uncoated silicon samples to determine the sample temperature as a function of heater temperature. As carbon coated sample we used a hard a-C:D film that was heated in a normal TE run to a maximum substrate holder temperature of $1300 \mathrm{~K}$ and held there for $30 \mathrm{~min}$. As we will see later, hydrogen is released and most of the carbon remains on the sample. This carbon layer doesn't change in consecutive annealing cycles. The emissivity of this carbon coated sample was determined by comparing the temperature evolution of a blank silicon sample with that of a carbon coated sample. From this comparison we estimated an emissivity of 0.73 for the carbon coated sample. At high temperature $(>1300 \mathrm{~K})$ the difference between the true sample temperature and the heater temperature can reach $350 \mathrm{~K}$.

These calibration measurements allow to determine the true sample temperature during normal TE runs. The uncertainty of the so-determined true sample temperature is estimated to be $\pm 15 \mathrm{~K}$ at about $1100 \mathrm{~K}$. This is inferior to the temperature determination in the glass oven. The reproducibility of the temperature calibration by this procedure is in general satisfactory. As a consequence of the slower heating of the sample compared with the heater, the real heating rate of the sample at higher temperature is about 10 to $20 \%$ lower than the experimentally set value. Consequently, the heating rates of the MBMS and oven setups are slightly different in the experiments reported here. This leads also to differences in the measured QMS intensities and complicates a quantitative comparison between QMS intensities measured in the MBMS and quartz oven setups.

The second strategy to determine the true sample temperature in the MBMS setup is to measure thermal effusion spectra for identical samples in the MBMS and quartz oven setups. In this case, we can use the temperature scale from the quartz oven spectra to determine the temperature of spectral features in the MBMS spectra. This allows an independent determination of the temperature scale in the MBMS setup. It is clear, that for a comparison of oven and MBMS spectra only data of non-reactive, recycling species can be used. In general the agreement of both methods was very satisfying.

\section{Investigation of redeposition}

The sticking of released species was investigated by thermo-desorption of the material redeposited along the glass tube. This was done by a systematic displacement of the oven after a normal TE run. First, the walls of the quartz glass tube were thoroughly cleaned by heating them to $1300 \mathrm{~K}$ for more than 90 minutes. To do this, the oven was first heated to $1300 \mathrm{~K}$ in the normal measurement position (centered around the closed end of the tube) and then moved in several steps towards the open end of the tube until the whole wall area that will be investigated later on was thoroughly annealed and cleaned. Then, after cooling down the oven, an a-C:D film was placed at the measurement position and a normal thermal effusion run up to the desired final temperature (in most cases $930 \mathrm{~K}$ ) was performed. After that, the oven temperature was held at that temperature until the pressure and the mass signal had sufficiently decreased $\left(\mathrm{p}<5 \times 10^{-8} \mathrm{~Pa}\right)$. In the following, the oven was moved by a defined distance (in most cases 1 or $2 \mathrm{~cm}$ ) towards the open end of the tube, so that a region that has been at room temperature during the effusion run was heated. This caused a strong increase of the chamber pressure due to release of redeposited material. After the pressure had again sufficiently decreased the oven was moved by another step. This procedure was repeated until the oven was moved by $12 \mathrm{~cm}$ from its original position.

\section{Sample preparation}

Amorphous deuterated carbon films (a-C:D) were produced in a capacitively coupled RF plasma setup using deuterated methane $\left(\mathrm{CD}_{4}\right)$ as working gas. The plasma chamber consists of a stainless steel vessel and was pumped to a base pressure in the $10^{-4} \mathrm{~Pa}$ range by a turbomolecular pump. Prior to deposition the substrate surfaces were cleaned by sputtering in an oxygen plasma followed by a hydrogen plasma (bias voltage $-300 \mathrm{~V}, 30 \mathrm{~min}$ each). The total methane $\left(\mathrm{CD}_{4}\right)$ pressure is kept at $2 \mathrm{~Pa}$ and the gas flow is adjusted by a mass flow meter at $20 \mathrm{sccm}$ (standard cubic centimeters per minute).

It's well known that the structure and physical properties of a-C:H respectively a-C:D films depend sensitively on the deposition conditions $[17,18]$. The properties of a-C:H films deposited from $\mathrm{CH}_{4}$ is by and large identical to those of a$\mathrm{C}: \mathrm{D}$ films deposited from $\mathrm{CD}_{4}$. The most important deposition parameter is the energy of ions impinging on the growing film surface during deposition [17-20]. At low ion energies $(<30 \mathrm{eV})$, soft, polymer-like films with high hydrogen content grow, while at higher ion energies hard and dense hydrocarbon films are deposited. The change of the physical properties of the films is a monotonic function of the ion energy. Typical hard, diamond-like amorphous carbon films with a hydrogen content of $\mathrm{H} /(\mathrm{H}+\mathrm{C}) \approx 0.3$ are produced at ion energies higher than $100 \mathrm{eV}[17,20]$. In order to deposit hard a-C:D films the silicon substrate is directly placed on the driven RF electrode which reached a self-bias voltage of $-300 \mathrm{~V}$. A second sample holder connected to ground potential is placed opposite to this electrode in a distance of $10 \mathrm{~cm}$ to deposit soft a-C:D films. Hard and soft films were deposited in the same deposition run on silicon wafers $100 \mathrm{~mm}$ in diameter. The film homogeneity across the wafer is better than 5\%. All samples used for thermal effusion measurements had the identical 
TABLE I: Physical properties of a-C:D films used in this publication. $\mathrm{n}$ and $\mathrm{k}$ are the real and imaginary part of the complex refractive index $(\hat{n}=n-i k)$ as measured by ellipsometry at $632.8 \mathrm{~nm}$. The deuterium content of the hard film was measured by ion-beam analysis; its density was calculated from the ion-beam data and the sample thickness. The deuterium content and density of the soft film were estimated based on published values $[17,20]$.

\begin{tabular}{|l|c|c|c|c|c|}
\hline sample & $\begin{array}{c}\text { film } \\
\text { thickness } \\
(\mathbf{n m})\end{array}$ & $\mathbf{n}$ & $\mathbf{k}$ & $\begin{array}{c}\text { Deuterium } \\
\text { content } \\
\mathbf{D} / \mathbf{D + C})\end{array}$ & $\begin{array}{c}\text { density } \\
\left(\mathbf{g ~ c m}^{-3}\right)\end{array}$ \\
\hline \hline hard a-C:D & 250 & 2.04 & 0.06 & 0.35 & 1.95 \\
\hline soft a-C:D & 180 & 1.55 & 0.001 & $\approx 0.50$ & $\approx 1.0$ \\
\hline
\end{tabular}

size of $10 \mathrm{~mm}$ by $10 \mathrm{~mm}$ (= coated area) and were cut from the identical wafer. Thus we can be sure that samples used in different TE runs are comparable.

To determine the optical properties and the sample thickness, a piece of sample was eroded in an oxygen plasma in an Electron Cyclotron Resonance (ECR) discharge measuring the film thickness change with an in-situ ellipsometer [20]. In addition, representative samples from the batch used for this study were investigated by ion-beam analysis to determine the $\mathrm{D}$ and $\mathrm{C}$ content of the samples. Details of the ion-beam analysis are described in [20]. The stoichiometry and optical properties of comparable a-C:H samples has been measured in the past [20]. The physical properties of the films used in this study are given in Table I. They are in good agreement with previous results $[17,20]$.

\section{RESULTS}

\section{A. Comparison of thermal effusion spectra of hard and soft a-C:D films}

Fig. 3 presents thermal effusion spectra (TE spectra) of a hard and a soft a-C:D film recorded in the MBMS configuration. Shown are mass signals of a number of representative masses as a function of temperature. The thickness of the hard a-C:D film was about $250 \mathrm{~nm}$ and that of the soft film $180 \mathrm{~nm}$ (see Table I). For the following comparison we should keep in mind that the total number of deuterium atoms in the samples is about the same while the total number of carbon atoms in the soft films is about half of that of the hard films. The TE spectra of the two films show significant differences. Firstly, effusion in the case of hard films starts at about $700 \mathrm{~K}$ and ends at 950 to $1000 \mathrm{~K}$ (with the exception of the mass 4 signal) whereas for soft films it starts at $600 \mathrm{~K}$ and ends at $800 \mathrm{~K}$. Secondly, the peak maxima occur at $870 \mathrm{~K}$ and $740 \mathrm{~K}$ for the hard and soft film, respectively. These two differences clearly show the lower thermal stability of soft films compared with hard films.

The assignment of the measured mass channels to specific
TABLE II: Assignment of selected mass channels to possible neutral mother molecules.

\begin{tabular}{|l|l|l|}
\hline mass (amu) & ion & mother molecule \\
\hline \hline 4 & $\mathrm{D}_{2}^{+}$ & $\mathrm{D}_{2}$ \\
\hline 18 & $\mathrm{CD}_{3}^{+}, \mathrm{H}_{2} \mathrm{O}^{+}$ & $\mathrm{CD}_{4}, \mathrm{H}_{2} \mathrm{O}$ \\
\hline 20 & $\mathrm{CD}_{4}^{+}$ & $\mathrm{CD}_{4}$ \\
\hline 28 & $\mathrm{C}_{2} \mathrm{D}_{2}^{+}, \mathrm{CO}^{+}$ & $\mathrm{C}_{2} \mathrm{D}_{2}, \mathrm{CO}$ \\
\hline 32 & $\mathrm{C}_{2} \mathrm{D}_{4}^{+}$ & $\mathrm{C}_{2} \mathrm{D}_{4}, \mathrm{C}_{2} \mathrm{D}_{6}, \ldots$ \\
\hline 34 & $\mathrm{C}_{2} \mathrm{D}_{5}^{+}$ & $\mathrm{C}_{2} \mathrm{D}_{6}, \mathrm{C}_{3} \mathrm{D}_{8}, \ldots$ \\
\hline 46 & $\mathrm{C}_{3} \mathrm{D}_{5}^{+}$ & $\mathrm{C}_{3} \mathrm{D}_{6}, \mathrm{C}_{4} \mathrm{D}_{8}, \ldots$ \\
\hline 50 & $\mathrm{C}_{3} \mathrm{D}_{7}^{+}$ & $\mathrm{C}_{4} \mathrm{D}_{10}, \mathrm{C}_{5} \mathrm{D}_{12}, \ldots$ \\
\hline 66 & $\mathrm{C}_{4} \mathrm{D}_{9}^{+}, \mathrm{C}_{5} \mathrm{D}_{3}^{+}$ & $\mathrm{C}_{4} \mathrm{D}_{10}, \mathrm{C}_{5} \mathrm{D}_{12}, \ldots$ \\
\hline
\end{tabular}

neutral hydrocarbon molecules is complicated by the fact that the cracking patterns for the long chain hydrocarbons become increasingly complex and many mass channels can have contributions from different neutral species. The identification and quantification of individual species is challenging and subject of ongoing work. A preliminary assignment of measured mass channels to neutral species is presented in Table II. The assignment is based on the fact that these ions have strong contributions in the respective cracking pattern of the molecules. The 4 amu mass channel stems from $\mathrm{D}_{2}$. The 20 amu mass channel can be solely attributed to $\mathrm{CD}_{4}$ since the cracking pattern of long chain hydrocarbons produces no contribution to mass 20 . In fact, this is the main reason why $\mathrm{a}-\mathrm{C}: \mathrm{D}$ films instead of a-C:H films have be used in this study. The dominant mass channel for $\mathrm{CH}_{4}$ is $16 \mathrm{amu}$. This overlaps with a cracking product of water from the background and, therefore, complicates the analysis. The same is true for $\mathrm{D}_{2}$ which can easily be distinguished from the residual contribution from $\mathrm{H}_{2}$ which is present in each vacuum system. All other mass channels have contributions from several mother molecules and cannot simply be assigned to one species.

Besides these different temperature ranges, the TE spectra differ significantly in the measured signal intensities. For a quantitative comparison we normalize the mass spectrometer intensities to the sample thickness (i.e., $\mathrm{cps} / \mathrm{nm}$ ). The maximum of mass $20\left(\mathrm{CD}_{4}\right)$ which is the dominant contribution in Fig. 3a (hard film) is about a factor of 10 higher than for the soft film shown in Fig. 3b (135 cps/nm compared with $13.2 \mathrm{cps} / \mathrm{nm})$. The intensity for $32 \mathrm{amu}$ is almost identical in both cases (23.4 and $21.6 \mathrm{cps} / \mathrm{nm}$ for hard and soft films, respectively). On the contrary, the normalized intensities for the masses 34, 46, 50, and 66 amu are all higher for the soft film. The ratios of the normalized intensities (soft/hard) for 34, 46, 50, and 66 amu are about 4, 25, 150, and 25. In particular mass 50 , which is a cracking product of $\mathrm{C}_{4} \mathrm{D}_{10}, \mathrm{C}_{5} \mathrm{D}_{12}$, and $\mathrm{C}_{6} \mathrm{D}_{14}$, is much higher for the soft film. The general trend is that the relative contribution of high molecular weight species is much higher for soft films compared with hard films. This is in agreement with published results for a-C:H films [21$24]$ and it is a consequence of the difference in microstructure 


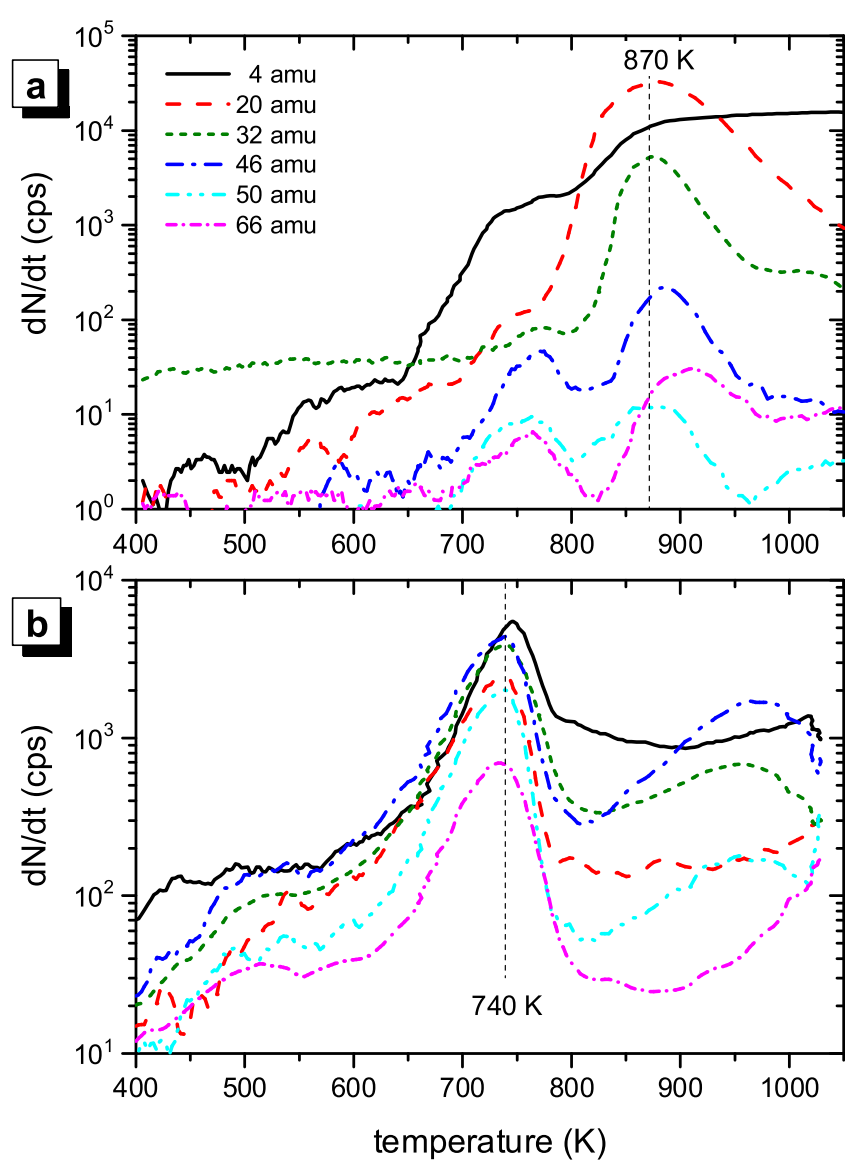

FIG. 3: Thermal effusion spectra of a (a) hard (thickness $252 \mathrm{~nm}$ ) and (b) soft (thickness $180 \mathrm{~nm}$ ) a-C:D film recorded in the MBMS configuration in the main chamber. Thermal desorption spectra are in logarithmic scale; please observe that different scales are used in a) and b). The temperature ramp was $15 \mathrm{~K} / \mathrm{min}$.

of the two different films.

In addition to the main peak, desorption from hard films (Fig. 3a) shows for the higher masses also a pre-peak at a temperature comparable to the desorption peak of soft films (Fig. 3b). This pre-peak has a much lower intensity than the main peak, but is clearly visible as a separate peak. For the soft film, we find a second broad desorption structure for the higher masses with a maximum at around $950 \mathrm{~K}$. This structure is attributed to an artefact of the measurement. High mass species desorbed from the sample may become redeposited on surrounding surfaces of the heater that are below the desorption temperature. With increasing heating time and increasing heater temperatures, these surfaces may reach desorption temperature and desorb the previously adsorbed species thus causing the second peak at high temperature. This interpretation is corroborated by the fact that this second peak for soft films is not observed in the oven setup (see Fig. 4b).

Similar measurements as those in Fig. 3 for the MBMS setup are shown in Fig. 4 for the oven setup. Qualitatively, the results measured in both setups are very similar. In particu-
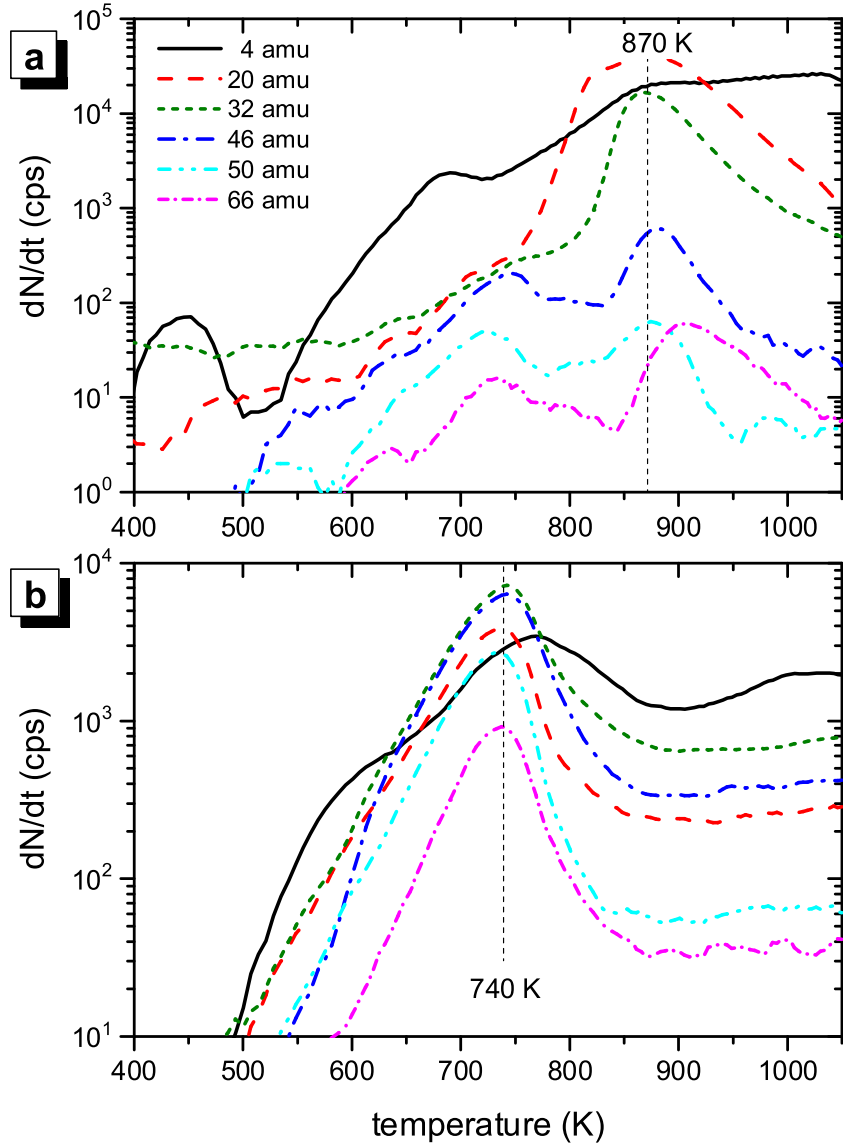

FIG. 4: Thermal effusion spectra of a (a) hard (thickness $252 \mathrm{~nm}$ ) and (b) soft (thickness $180 \mathrm{~nm}$ ) a-C:D film recorded in the UHV oven (glass tube). Thermal desorption spectra are in logarithmic scale; please observe that different scales are used in a) and b). The temperature ramp was $15 \mathrm{~K} / \mathrm{min}$.

lar, the temperature ranges and peak shapes of the TE spectra for the two different films are in very good agreement. But significant quantitative differences are observed. The differences to the MBMS setup occur with respect to the relative contributions of different masses. Although the QMS is the identical instrument in both cases, QMS intensities measured in the oven and MBMS setup cannot directly be compared (as discussed in Sect. II B 2). But, we can compare at least relative intensities. For this comparison, we relate the measured QMS intensities to the mass 20 signal of each spectrum. For the experiments in the MBMS setup, the relative signal of the high mass species ( $\geq 34 \mathrm{amu}$ ) is smaller than 0.02 for the hard film, while it is of the order of 1 for the soft film, so that the ratios of the normalized intensities between soft and hard films range from 44 to 1500 . However, for the experiments in the oven setup, these ratios are only between 16 and 500, i.e., they are about a factor of 2 to 3 lower than in the MBMS setup.

Fig. 5 shows for two identical, soft a-C:D samples relative intensities of 4 different masses measured in the MBMS and oven setup, respectively. Here, for normalization we in- 

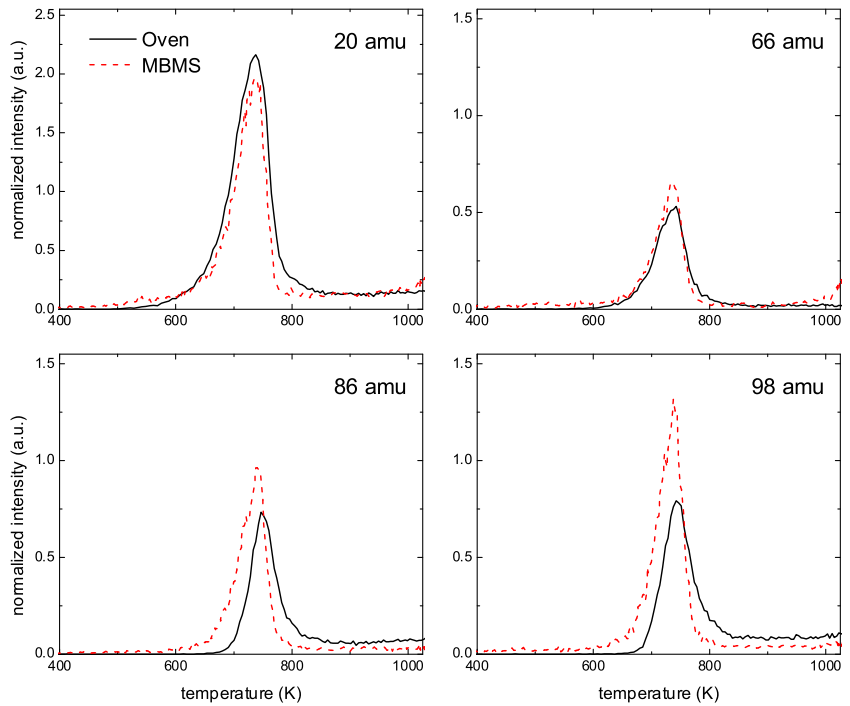

FIG. 5: Comparison of thermal effusion spectra of soft a-C:D films measured in the MBMS and UHV oven (glass tube) setups. The following masses are shown: a) $20 \mathrm{amu}$, b) $66 \mathrm{amu}$, c) $86 \mathrm{amu}$, and d) $98 \mathrm{amu}$. The data are normalized to the respective integral over the $20 \mathrm{amu}$ peak as described in the text. The data are plotted in arbitrary units, but on a common scale for all 4 figures, so that the relative intensities can be compared. Please observe that the scale in Figs. $b$ to $d$ is slightly enlarged. The peak shape and position of the normalized 20 amu peak (a) is in excellent agreement for both measuring setups. The peaks for 86 and 98 amu are shifted towards higher temperatures for the measurements in the oven setup.

tegrated the desorption peaks in the range from 600 to $850 \mathrm{~K}$ and set the integral of the 20-amu signal to 1 . All other peak integrals for the same setup are normalized relative to this 20amu signal. Because the heating rates in both setups are not fully identical and for the MBMS setup not completely linear (see Sect. II B 2) we performed the integration on the experimental time scale and not on the temperature scale. As a consequence, the areas under the two 20-amu curves are not identical if plotted on the temperature scale (as in Fig. 5), but this does not influence the general conclusions. The peak shape and position of the $20 \mathrm{amu}\left(\mathrm{CD}_{4}\right)$ peaks are almost identical in both cases (Fig. 5a). The difference between the signal of the MBMS and oven measurement becomes larger with increasing molecular weight of the species (masses 66, 86, and 98 amu in Fig. 5b to d). A quantitative comparison of the difference in measured relative signal intensities is presented in Table III for 8 representative masses. In addition, the ratio between the relative signal intensities is shown.

\section{B. Redeposition of thermally released products}

Redeposition was measured by moving the oven to a new position after a normal TE run as described in Sect. II C. Figure 6 shows the $\mathrm{CD}_{4}$ signal measured with the mass spectrometer and the pressure gauge reading in the main chamber as a function of time during two separate experiments in the
TABLE III: Normalized (to mass 20) peak integrals (integration range 600 to $850 \mathrm{~K}$ ) of selected masses measured for soft a-C:D films in the MBMS and oven setup.

\begin{tabular}{|l|r|r|r|}
\hline $\begin{array}{l}\text { mass } \\
(\mathrm{amu})\end{array}$ & $\begin{array}{r}\text { oven } \\
\text { (rel. int.) }\end{array}$ & $\begin{array}{r}\text { MBMS } \\
\text { (rel. int.) }\end{array}$ & $\begin{array}{r}\text { ratio } \\
\text { (oven/MBMS) }\end{array}$ \\
\hline \hline 20 & 1.00 & 1.00 & 1.00 \\
\hline 34 & 0.74 & 0.83 & 0.89 \\
\hline 46 & 1.67 & 1.83 & 0.91 \\
\hline 50 & 0.63 & 0.73 & 0.85 \\
\hline 66 & 0.21 & 0.30 & 0.69 \\
\hline 74 & 0.41 & 0.50 & 0.82 \\
\hline 82 & 0.23 & 0.38 & 0.60 \\
\hline 86 & 0.25 & 0.42 & 0.60 \\
\hline 98 & 0.32 & 0.55 & 0.58 \\
\hline
\end{tabular}

oven using a hard and a soft film, respectively. In each experiment first a normal TE run was performed and then the oven was shifted in steps of $2 \mathrm{~cm}$. Identical samples as for the TE runs shown in Figs. 3 and 4 were used. The first peak in Fig. 6 corresponds to desorption from the sample for a heating rate of $15 \mathrm{~K} / \mathrm{min}$ up to $930 \mathrm{~K}$ (i.e., this is the normal effusion peak). After reaching the temperature of $930 \mathrm{~K}$ the oven was held constant at that temperature. The second peak corresponds to desorption from the walls of the glass tube after the first displacement by $2 \mathrm{~cm}$, and the following peaks to the consecutive displacements of the oven. In the following we will denominate the first peak as primary peak and the following desorption peaks measured after each displacement step as secondary peaks.

The inset in Fig. 6 shows the reading of the cold cathode gauge in the main vacuum chamber. This reading is correlated with the total pressure, but due to the contribution of many different species with largely varying sensitivity factors this value cannot easily be converted to the total pressure. For the first peak we find a much higher signal for the hard film than for the soft film. This is in accordance with the results of the mass resolved spectra presented in Figs. 3 and 4. In contrast, the signals from the secondary peaks are much higher for the soft film. This clearly proves that the redeposition fraction is much higher for soft films than for hard films. In the case of the hard film the integrated area under all 6 secondary peaks of the pressure reading corresponds to about $6 \%$ of the area of the primary peak and to $42 \%$ in the case of the soft film.

The difference between soft and hard films is even larger if the $\mathrm{CD}_{4}$ (20 amu) signal is considered. Although the peak maximum of the hard film is about a factor of 20 higher than that of the soft film, the secondary peaks of the hard film are about a factor of 2 smaller than those of the soft film. For the hard film, the integrated area under all 6 secondary peaks corresponds to about $0.2 \%$ of the area of the primary peak and to $70 \%$ for the soft film. This strong difference between the total pressure and mass 20 is again an indication that other species have to contribute dominantly to the released species flux. 


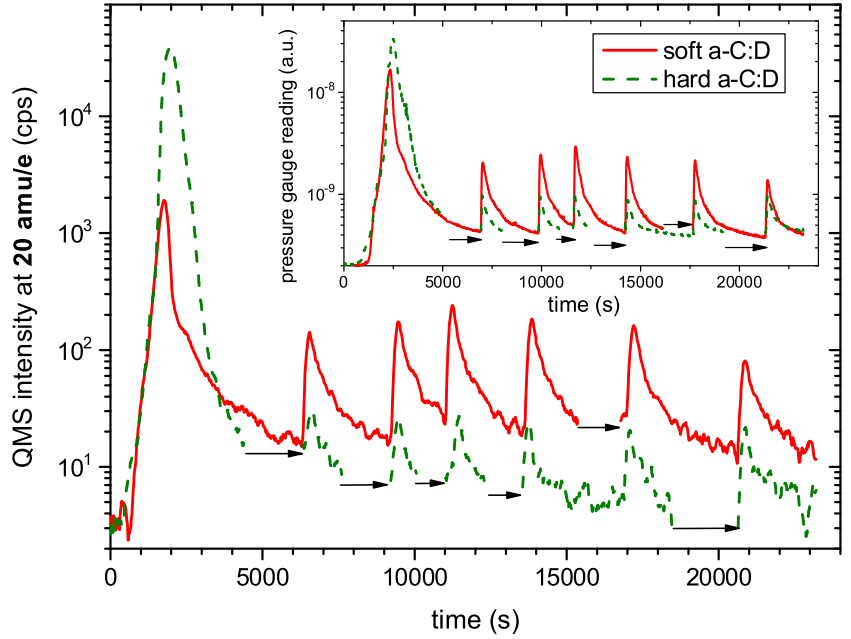

FIG. 6: TE spectra for mass 20 of a soft and hard film measured in the oven setup in the usual measurement position (primary peak) and after consecutive shifts of the oven by $2 \mathrm{~cm}$ (secondary peaks). The inset shows the cold cathode gauge reading for the identical experiment. Because the time scales of the two experiments are not identical, the positions of the secondary peaks have been shifted accordingly to allow a comparison of the intensities of the peaks. These shifts are indicated by the arrows in the figure.

Similar data as for mass 20 in Fig. 6 are shown for mass 46 in Fig. 7. In contrast to mass 20 (Fig. 6), the peak maximum of the primary peak of the hard film is smaller than that of the soft film. The double peak structure of the hard film are the pre- and main peaks that were already shown in Figs. 3a and $4 \mathrm{a}$. The pre-peak for the hard film is particularly strong for mass 46. Also in striking contrast to mass 20, the ratio of the integrated area under all 6 secondary peaks to the primary peak is comparable for mass 46 . The area under the secondary peaks corresponds to $26 \%$ for the hard film and to $35 \%$ for the soft film.

\section{DISCUSSION}

Comparing TE spectra measured in the MBMS (Fig. 3) and oven (Fig. 4) setup we found significant quantitative differences. For the measurements in the oven setup the relative contribution of the high mass species is about a factor of 2 to 3 lower than in the MBMS setup. We interpret this as a significantly lower probability for the high molecular weight species to reach the mass spectrometer if released in the oven as compared with the MBMS setup. We assume that this is due to an increased probability to stick to the walls. This is a plausible explanation, because the species released in the oven setup have to survive many wall collisions before they can reach the ionizer of the mass spectrometer. This is also in agreement with the investigations regarding redeposition which were presented in Sect. III B.

The large difference between hard and soft films can be understood on the basis of prior knowledge on the microstructure

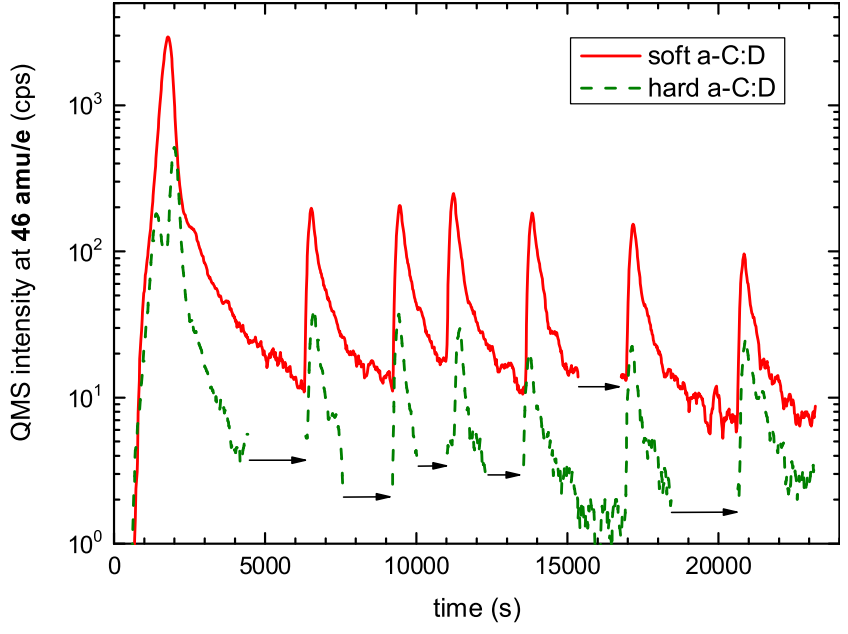

FIG. 7: Same as figure 6 but for 46 amu.

of a-C:H films. It is, for example, well known that they contain different amounts of hydrogen [17-20, 24]. While hard films have typical hydrogen concentrations of $30 \%$, soft films exhibit hydrogen concentrations of $50 \%$ and more. More hydrogen in the films leads to more terminal hydrocarbon groups which have only one remaining bond to the carbon network. These terminal groups can be released during thermal decomposition. With further increasing hydrogen content these terminal groups may get longer thus leading to a larger fraction of long chain, i.e., high molecular weight, hydrocarbon species. Although this is a reasonable explanation, it has to be kept in mind that only little is known about these processes and from the available data no conclusions can be drawn about the basic release mechanisms.

In Fig. 5 we compared the peak shapes of normalized TE spectra measured in both setups and compiled a quantitative comparison of the normalized peak integrals in Table III. For all masses other than 20 amu (which was used for normalization) the peak integral of the oven measurement is lower than that of the MBMS measurement. For masses 34, 46, 50, and 74 the ratio varies between 0.82 and 0.91 which means that about 10 to $20 \%$ of the species released in the oven are not detected by the mass spectrometer. The difference is even larger for masses $66,82,86$, and 98 . For these species the loss of intensity lies between 30 and $40 \%$. For 86 and 98 amu not only the peak integral is about $40 \%$ lower but also the peak is shifted to higher temperature in the oven measurement. We assume that both effects can be explained by an enhanced sticking of some of the high molecular weight species to the wall of the glass tube. Some of these species are permanently lost at the wall and are responsible for the loss of intensity. Others reach the mass spectrometer with some delay due to many adsorption desorption steps experienced during transport to the mass spectrometer.

To test the hypothesis of sticking to the walls of the quartz glass tube, we investigated the amount of redeposited material directly (see Sect. III B). TE spectra for 20 and $46 \mathrm{amu}$ and the total pressure in the main chamber are presented in 
Figs. 6 and 7. Each movement of the oven gives rise to an increase of the total pressure. The only possible interpretation for this is, that species released from the sample redeposit on the non heated parts of the quartz glass tube. The fact that a $\mathrm{CD}_{4}$ signal is observed in the secondary peaks of Fig. 6 is remarkable. $\mathrm{CD}_{4}$ is a stable molecule and does not adsorb on the quartz glass surface at $300 \mathrm{~K}$. This is in agreement with results for the hard film. Mass 20, which is solely attributed to $\mathrm{CD}_{4}$, constitutes the dominant TE peak for hard films (Figs. 3a and $4 \mathrm{a}$ ) and the total redeposition is only about $0.2 \%$ (Fig. 6). So we have to conclude that $\mathrm{CD}_{4}$ measured in the secondary peaks is not deposited as a $\mathrm{CD}_{4}$ molecule, but is a reaction product generated during the following desorption step. This indicates that the released species spectrum is not identical to the adsorbed species spectrum. Hence, we have to conclude that in each desorption step new species are produced from the adsorbed species. This is a question that has to be studied in more detail in future investigations. Furthermore, this effect rules out any attempt to quantify redeposition of individual species, e.g., determining the sticking coefficient, from such kind of measurements. We only can compare relative signals measured in the MBMS and oven setups as shown in the previous section (Fig. 5).

A further interesting detail of the oven displacement measurements is the observed behavior of the maximum value of each secondary peak. Assuming a thickness profile of the redeposited film that decays monotonously with increasing distance from the end of the hot zone, we would anticipate that the maximum value of the secondary peaks decreases monotonically. This is, however, not what is observed. The maximum of the first 3 secondary peaks increases and then for the following displacements it decreases again. This behavior, which is also observed for the integral over the secondary peaks, is not yet understood, but we assume that it is due to a non-monotonic thickness profile. A possible explanation for that could be a slight heating of the inner surface of the quartz glass tube due to thermal radiation from the oven which could lead to a lower deposition close to the oven. An explanation of this effect requires further investigations.

Redeposition plays a measurable but small role for hard a$\mathrm{C}: \mathrm{D}$ films, but it is of paramount importance for soft films. It should be emphasized that the value of $42 \%$ for redeposition from soft films deduced from the analysis of the total pressure corresponds to the first $12 \mathrm{~cm}$ of the quartz glass tube only. A substantial fraction of the released material will be redeposited on the following $17 \mathrm{~cm}$ of the glass tube and $35 \mathrm{~cm}$ of the stainless steel pipe between main chamber and glass tube. We further have to assume that a measurable fraction of the species reaching the main chamber may deposit on the main chamber walls. This means that in our experimental geometry the total redeposition of species released in the oven setup from soft films can easily exceed $50 \%$. With other words, this means that less than $50 \%$ of the species released from the sample have the chance to reach the QMS. Naturally, the redeposition fraction will strongly depend on the sticking probability of the individual species. As the preceding discussion is mainly based on the $\mathrm{CD}_{4}$ signal and the measurement of the pressure which are both dominantly due to recycling species, we conclude that the average redeposition probability for reactive species is much higher than $50 \%$. So we conclude that the redeposition of material on the walls of the quartz glass tube is the main reason for the much lower signal measured in the oven setup compared with the MBMS setup.

\section{CONCLUSIONS}

Thermally induced decomposition of hard and soft amorphous hydrocarbon films was investigated by thermal effusion spectroscopy in the experimental setup TESS.

Thermal decomposition of a-C:D films leads to release of molecular hydrogen and a large variety of hydrocarbon molecules. The spectrum of released species depends strongly on the physical properties of the used a-C:H(D) films, in particular on the hydrogen content. Comparable results have been found for $\mathrm{a}-\mathrm{C}: \mathrm{H}$ films [21-24] so that we can assume that the thermal decomposition is not significantly influenced by type of hydrogen isotope in the film. In this study, two archetypes of a-C:D films were used. A typical hard film with a hydrogen content of about $30 \%$ and a soft film with $50 \%$. Soft a-C:D films exhibit a desorption maximum at $\mathrm{T} \approx 740 \mathrm{~K}$ while hard films have their maximum at $\mathrm{T} \approx 870 \mathrm{~K}$. For hard films the dominantly released species are $\mathrm{D}_{2}$ and $\mathrm{CD}_{4}$. On the contrary, the product spectrum of soft films is dominated by long chain hydrocarbons. $\mathrm{C}_{2} \mathrm{D}_{y}$ and $\mathrm{C}_{3} \mathrm{D}_{y}$ dominate, but significant contributions of $\mathrm{C}_{4} \mathrm{D}_{y}$ and $\mathrm{C}_{5} \mathrm{D}_{y}$ were also found.

The higher thermal stability and the different product spectrum of hard films can be understood on the basis of prior knowledge of the films microstructure. More hydrogen in the films leads to more terminal hydrocarbon groups. These terminal groups can be released during thermal decomposition. With further increasing hydrogen content these terminal groups may get longer thus leading to a larger fraction of long chain hydrocarbon species.

Comparing spectra recorded in a molecular-beam-massspectrometry setup with spectra recorded by the same mass spectrometer but from an UHV oven in a remote location, we found that released species have a certain probability to stick to the chamber walls. Redeposition plays a small role for hard a-C:D films, but it is of paramount importance for soft films. This redeposition was studied qualitatively in a dedicated experiment by investigating the re-desorption of redeposited material. We conclude that redeposition is mainly due to sticking of high molecular weight species. An interesting aspect is the fact that obviously new species are formed during re-desorption of redeposited material. From the redeposited fraction of material we estimated a total redeposition probability of more than $50 \%$ for species released from soft films for our experimental conditions.

This strong tendency for redeposition limits the applicability of thermal methods for hydrogen removal from fusion devices. Thermal methods are applicable to hard a-C:H(D) films, because the spectrum of released species is dominated by the stable products $\mathrm{H}_{2}$ and $\mathrm{CH}_{4}$ (or comparable species formed with other hydrogen isotopes) which do not redeposit on cooler wall areas. Soft, hydrogen-rich films decompose 
into a rich spectrum of species which partially tend to stick to cooler wall areas. As a consequence, local heating will remove such films from the heated area, but the material will not be transported to the vacuum pumps but redeposit somewhere else in the vacuum vessel. Therefore, to demonstrate the feasibility of thermal methods it is not sufficient to demonstrate local removal, but to prove that the mobilized inventory can be really removed from the vacuum vessel.
Acknowledgement

One of us (E.S.) would like to thank EURATOM Association for funding the stay at IPP Garching.
[1] G. Federici, P. Andrew, P. Barabaschi, J. Brooks, R. Doerner, A. Geier, A. Herrmann, G. Janeschitz, K. Krieger, A. Kukushkin, et al., J. Nucl. Mater. 313-316, 11 (2003).

[2] J. Paméla, G. Matthews, V. Philipps, R. Kamendje, and JETEFDA Contributors, J. Nucl. Mater. 363-365, 1 (2007).

[3] A. von Keudell, C. Hopf, T. Schwarz-Selinger, and W. Jacob, Nucl. Fusion 39, 1451 (1999).

[4] C. Hopf, K. Letourneur, W. Jacob, T. Schwarz-Selinger, and A. von Keudell, Appl. Phys. Lett. 74, 3800 (1999).

[5] C. Hopf, T. Schwarz-Selinger, W. Jacob, and A. von Keudell, J. Appl. Phys. 87, 2719 (2000).

[6] J. P. Coad, N. Bekris, J. D. Elder, S. Erents, D. Hole, K. Lawson, G. Matthews, R. Penzhorn, and P. Stangeby, J. Nucl. Mater. 290-293, 224 (2001).

[7] M. Rubel, J. P. Coad, N. Bekris, S. Erents, D. Hole, G. Matthews, R. Penzhorn, and Contributors to EFDA-JET work programme, J. Nucl. Mater. 313-316, 321 (2003).

[8] M. Mayer, V. Rohde, A. von Keudell, and ASDEX Upgrade Team, J. Nucl. Mater. 313-316, 429 (2003).

[9] M. Mayer, A. von Keudell, V. Rohde, P. Coad, and JET-EFDA contributors, in Proceedings of the 30th EPS Conference, edited by R. Koch and S. Lebedev (European Physical Society, 2003), vol. 27A of European Conference Abstracts, pp. O-2.6A.

[10] V. Rohde, M. Mayer, J. Likonen, R. Neu, T. Pütterich, E. Vainonen-Ahlgren, and ASDEX Upgrade Team, J. Nucl. Mater. 337-339, 847 (2005).

[11] G. Counsell, P. Coad, C. Grisolia, C. Hopf, W. Jacob, A. Kirschner, A. Kreter, K. Krieger, J. Likonen, V. Philipps, et al., Plas. Phys. Control. Fusion 48, B189 (2006).
[12] W. Jacob, J. Nucl. Mater. 337-339, 839 (2005).

[13] C. Skinner, N. Bekris, J. P. Coad, C. Gentile, and M. Glugla, J. Nucl. Mater. 313-316, 496 (2003).

[14] N. Bekris, J. Coad, C. Skinner, C. Gentile, E. Damm, and W. Nägele, J. Nucl. Mater 367-370, 1254 (2007).

[15] K. Gibson, G. Counsell, C. Curran, M. Forrest, M. Kay, and K. Watkins, J. Nucl. Mater. 337-339, 565 (2005).

[16] A. Widdowson, J. Coad, N. Bekris, G. Counsell, M. Forrest, K. Gibson, D. Hole, J. Likonen, W. Parsons, T. Renvall, et al., J. Nucl. Mater. 363-365, 341 (2007).

[17] W. Jacob, Thin Solid Films 326, 1 (1998).

[18] J. Robertson, Mat. Sci. Eng. Reports 37, 129 (2002)

[19] M. Weiler, S. Sattel, T. Giessen, K. Jung, H. Ehrhardt, V. S. Veerasamy, and J. Robertson, Phys. Rev B 53, 1594 (1996).

[20] T. Schwarz-Selinger, A. von Keudell, and W. Jacob, J. Appl. Phys. 86, 3988 (1999).

[21] C. Wild and P. Koidl, Appl. Phys. Lett. 51, 1506 (1987).

[22] J. Ristein, R. T. Stief, L. Ley, and W. Beyer, J. Appl. Phys. 84 3836 (1998).

[23] W. Jacob, C. Hopf, A. von Keudell, and T. Schwarz-Selinger, Hydrogen Recycling at Plasma Facing Materials (Kluwer Academic Publishers, Dordrecht, 2000), chap. Surface Loss Probabilities of Hydrocarbon Radicals on Amorphous Hydrogenated Carbon Film Surfaces: Consequences for the Formation of Redeposited Layers in Fusion Experiments, pp. 331-337.

[24] T. Som, M. Malhotra, V. Kulkarni, and S. Kumar, Physica B 355, 72 (2005) 